\title{
EI Juego como recurso fundamental para la resolución de conflitos
}

\section{Play as a fundamental resource for conflict resolution}

DOI: $10.46932 / \mathrm{sfjdv2n5-063}$

Received in: Oct 1st, 2021

Accepted in: Dec 30th, 2021

\author{
María Del Carmen Vivero Riquelme \\ Grado en Educación Infantil y Primaria \\ RG FORMACIÓN \\ Calle Gabriel García Márquez, 3. 30508, Murcia. \\ E-mail: investigacionrgformacion@gmail.com
}

\begin{abstract}
RESUMEN
Este proyecto surge con la idea de dar una respuesta a los distintos conflictos que surgen en un aula de alumnos en edad infantil, problemas para los que pretendemos aportar una solución basada en el diálogo y la mediación pacífica entre los alumnos y su educador que, apoyándose en el juego como estrategia didáctica e indispensable de la educación infantil, podrá superar los distintos conflictos presentes en esta etapa escolar. Por lo tanto, la principal pregunta que pretendemos responder con este trabajo es la siguiente: ¿Cómo podemos resolver un conflicto a través del juego?

Para responder esta cuestión, hemos diseñado un proyecto novedoso e interdisciplinar que incluye una serie de actividades que permitirán al docente evaluar y analizar la evolución de sus alumnos a lo largo del curso-etapa.
\end{abstract}

Palabras clave: resolución de conflictos, juegos, educación en valores, trabajo cooperativo.

\section{ABSTRACT}

This project arises with the idea of giving an answer to the different conflicts that arise in a classroom of children, problems for which we intend to provide a solution based on dialogue and peaceful mediation between students and their educator that, relying on the game as a didactic and indispensable strategy of early childhood education, can overcome the various conflicts present in this school stage. Therefore, the main question we intend to answer with this work is the following: How can we solve a conflict through play?

To answer this question, we have designed a novel and interdisciplinary project that includes a series of activities that will allow the teacher to evaluate and analyze the evolution of their students throughout the course-stage.

Keywords: conflict resolution, games, values education, cooperative work.

\section{INTRODUCCIÓN}

Resulta extraño combinar dos conceptos tan dispares como 'juego' y 'conflicto'. El término ‘juego' se relaciona a algo lúdico, creativo o divertido, mientras que cuando nos referimos a la idea de 'conflicto' suele estar asociada a algo negativo. Esto último lo consideramos una idea equivocada, pues 
pensamos que el conflicto es un proceso natural por el que pasamos todos, proporcionándonos un aprendizaje que nos ayudará a resolver nuestros problemas y a conocer a las personas con las que nos cruzamos a lo largo de nuestra vida, además de los rasgos de su personalidad y de la nuestra propia.

La elección del tema nace de la observación y de las vivencias desarrolladas en un centro educativo ubicado en Murcia, concretamente, en una clase de segundo ciclo de Educación Infantil formada por 24 alumnos y caracterizada por su gran diversidad.

La sala en la que se imparte clase está dividida en varios espacios, entre los que se encuentran el rincón de lectoescritura, el lógico-matemático y el de juego libre, entre otros. Pero, sobre todo, en este estudio vamos a centrarnos en la importancia del espacio conocido como asamblea, que es el lugar elegido para la comunicación entre él maestro y sus alumnos. En ella, se resuelven los conflictos que se van sucediendo en el aula de infantil. Además, de los rincones tradicionales nombrados anteriormente, durante mis prácticas curriculares también pude observar la existencia de nuevos espacios como el llamado 'Escucha y Habla', reservado para la resolución de conflictos de manera más íntima y autónoma que la asamblea.

En algunas ocasiones, los profesores pecamos de esquivar los conflictos para no crear un mal ambiente o generar un problema en clase, centrándonos en la enseñanza de los saberes clásicos como las letras o las matemáticas, a cuyo aprendizaje siempre se les ha dedicado más tiempo que a la transmisión de valores como el perdón, la igualdad o el respeto. Con esto quiero decir, que considero necesaria la orientación de la educación hacia un modelo que no solo se encargue de enseñar conocimientos académicos y, para ello, hemos incluido algunas actividades que ayudarán a ello.

Ejemplos de esta manera de educar, cada vez está más implantada en las aulas, los encontramos en actividades que hemos incluido en el proyecto, como la conocida como Scape Room. Este tipo de juegos, que despiertan la curiosidad y atención de los alumnos, son recursos de entretenimiento y cooperación aptos para que los pequeños aprendan a empatizar con los demás.

La resolución de conflictos es tan necesaria en nuestra sociedad que se encuentra regulada, mediante documentos legislativos vigentes, en el sistema educativo español. Algunos de los principios relacionados con la resolución de conflictos son los siguientes:

Real Decreto 1630/2006, de 29 de diciembre, por el que se establece las enseñanzas mínimas de segundo ciclo de Educación Infantil en el artículo 4.

Los objetivos propuestos, en esta norma, para la resolución de conflictos están orientados a enseñar a los niños a que se comuniquen con sus compañeros, obteniendo unas normas básicas de comportamiento. También destaca la importancia de trabajar en equipo para resolver los problemas de forma sosegada mediante el diálogo, atendiendo a las necesidades de los alumnos de manera personalizada. 


\section{EL CONFLICTO}

Tras una amplia búsqueda documental sobre este término, caben destacar las siguientes definiciones:

Castells (citado en Narejo y Salazar, 2002) considera que el conflicto es un acto que ocurre frecuentemente en la sociedad y en las interacciones entre dos o más personas. Jares (referenciado en Laponni, 2013) amplió esta definición añadiendo las causas del conflicto, indicando que su aparición en personas o grupos sociales "está determinada por la incompatibilidad, ya sea personal o en los valores o intereses que sostienen" (p. 92).

Por su parte, Ruiz (2002) indicó que los conflictos se producen de forma natural a través del acto de comunicación, ayudando a adquirir multitud de saberes como el desarrollo personal del sujeto y la formación. Visión parecida de este concepto es la que tiene Rodríguez, que dijo que "el conflicto es un hecho básico de la vida y una oportunidad constante para aprender, es parte natural y orgánica de la interacción social; y el hecho de aprender a mirarlo, entenderlo y analizarlo puede ayudarnos a forjar respuestas más efectivas y productivas" (2004, p. 145). Estas definiciones del conflicto nos ayudan a alejarlo de la imagen tan negativa que, en un principio, todos asociamos al término.

Según Lapponi (2013), el conflicto es un conjunto de acciones por el que debaten unas personas con otras para alcanzar un objetivo común. Al igual que hemos visto en la anterior definición de Jares, piensa que suele tener su origen a la hora de tener una idea distinta o contraria a sus propias necesidades e intereses.

Pero si hay una definición completa y que apela al papel instructor que tienen los conflictos, es la que aportó Torrego (2008):

"El conflicto se puede interpretar como una realidad socialmente construida y desarrollada a través de la comunicación por parte de los protagonistas en un contexto socio histórico determinado que acaba afectando a la interpretación y la conducta de las personas implicadas” (p. 373).

Tras estas definiciones, podemos comprobar que el conflicto es una situación presente en todos los ámbitos de nuestra vida, y el entorno escolar no está exento de dicha confrontación.

\section{DIMENSIÓN ESCOLAR O EDUCATIVA DEL CONFLICTO}

Es imposible que no nos crucemos con enfrentamientos a lo largo de nuestra trayectoria educativa. De hecho, autores como García (1998), afirman que los conflictos siguen teniendo mucha relevancia en el día a día de los centros escolares.

Ahondando un poco más en las disputas presentes en el aula encontramos a Allport (citado en Hamodi y Jiménez, 2018), que comparte que en los espacios en los que coinciden los alumnos surgen 
numerosos conflictos. Esto se debe a que existe una gran diversidad dentro del alumnado, es decir, cada uno muestra una personalidad diferente y la relación de cada niño con su unidad familiar es distinta y le condiciona a la hora de comunicarse con sus iguales. Para la resolución de estos enfrentamientos, autores como Gil, Redondo y Arribas (citados en Rodriguez y Ordóñez, 2017) resaltaron la importancia del espacio dedicado a la asamblea o reunión de alumnos, añadiendo que es una zona de comunicación óptima para solventar los conflictos anteriores, sucedidos por ejemplo durante el recreo.

En lo relativo a la actitud del docente frente a estos problemas, Ramos (2017) afirmó que los conflictos se pueden solventar desde distintas perspectivas, pero cada individuo tiene su propia manera para solucionarlo. Aspectos como la inteligencia emocional, la inteligencia sociocognitiva, la moral y el futuro resultan claves a la hora de hacer frente a los conflictos.

Como ya hemos dicho, la gran diversidad del alumnado o las relaciones familiares pueden ser motivo de confrontación dentro del espacio escolar, pero Serrano y Guzmán (2011) añaden algunas razones más, como la organización del centro o los propios maestros.

\section{EDUCACIÓN EN VALORES}

Una de las formas más efectivas para minimizar los inevitables conflictos en las aulas es la educación en valores. La Asociación Mundial de Educadores Infantiles (Amei Waece) señala que "hasta ahora se ha considerado que la educación en valores tiene una gran relevancia en esta etapa, pues conjuga un enfoque globalizado del mundo que los niños y niñas en ese momento tienen de la vida que les rodea" (citada en Díaz y Cuenca, 2015, p. 1094). Tras dicha definición, podemos entrever que una de las características de este tipo de formación es hacer a los niños partícipes en el mundo.

Según Marín y Sánchez, “educar es hacer que el educando ponga los valores en su vida, que los conozca, los aproveche, los estime y los haga crecer" (2015, p. 1096). Esta forma de enseñar, tan a largo plazo en la edad infantil, resulta tarea difícil para unos maestros que deberán transmitir con paciencia los mensajes oportunos para contribuir de manera positiva a la educación de los alumnos.

Dentro de este tipo de educación destaca la figura del profesor. Según Touriñan (2006), la labor de un maestro a la hora de la enseñanza de valores no se trata de someter al educando a aprendizajes sobre los saberes, sino que el niño debe descubrir y experimentar que le proporcionan esos conocimientos. Es decir, para que un alumno adquiera un valor debe comenzar comprobando los buenos resultados que les aporta esa conducta.

Podríamos definir este tipo de educación de la manera en que lo hizo Chillón (1996), que aconsejó a los docentes que los alumnos: 


\begin{abstract}
"Tomen parte activa en todas las decisiones importantes de aula, que adquieran ciertas responsabilidades, que participen en la elaboración de normas que rigen la actividad escolar, en su regulación y en el establecimiento de sanciones de incumplimiento. De esta manera van tomando progresivamente conciencia del sentido de la responsabilidad, de lo que supone la obediencia o no a unas normas y las consecuencias positivas o negativas de la acción”. (p. 26).
\end{abstract}

Pero encontramos a otros investigadores disconformes con esta moderna idea de educación. Uno de ellos es Descalzi, que piensa que "la educación de los valores fracasa, entre otros motivos, porque carece de un fundamento teórico suficiente" (1997, p. 123). Entre otras teorías que respaldan esta afirmación encontramos la de Sánchez y Vallejo (2006), que piensan que educar en valores es una labor compleja, ya que cada individuo lleva consigo una serie de creencias, intereses y necesidades. En ocasiones, los maestros y familias prescinden de la educación en valores, ya que conforma una educación compleja de explicar y muy personalizada.

\title{
5 ESTRATEGIAS PARA LA RESOLUCIÓN DE CONFLICTOS
}

En la construcción de estrategias resolutorias resalta la figura del educador, que debe tener aptitudes para "reconvertir el conflicto, regularlo de forma creativa, ser capaz de desarrollar el tratamiento positivo del mismo y dar lugar a la construcción de experiencias significativas, a través de procesos como pueden ser la mediación y la negociación” (Narejo, 2002, p.2). Según la perspectiva que obtenemos de este autor, es muy importante prestar atención al acto de comunicación para solucionar un conflicto, es decir, escuchar ambas partes y encontrar, de manera acordada por el grupo implicado, una solución. En este proceso, destacan la negociación y la mediación como elementos pacificadores.

La gran mayoría de autores coinciden en situar al educador como elemento clave de conciliación. Domínguez (2013) afirma que un educador social debe modelar y estudiar aspectos relacionados con la educación emocional para poder hacer frente a la resolución de conflictos de manera efectiva. Su misión es examinar el motivo de los conflictos que surgen en el aula. Visión parecida tiene Rodríguez (2004), que piensa que el profesorado debe enseñar a los alumnos destrezas comunicativas, como la manifestación clara de ideas y sentimientos, mostrando realmente que nos preocupamos por los demás mediante las relaciones interpersonales que establecemos con otra persona. Según la autora, la adquisición de dichas técnicas ayudará a la suavización de los conflictos que surjan entre los niños.

También cabe destacar el aspecto temporal de la resolución de conflictos. Serrano y Guzmán (2011) opinan que no es una acción que se realiza para solucionar el problema de inmediato, sino que requiere de un tiempo para acabar de forma absoluta. "Los conflictos pueden desarrollar estrategias de resolución inefectivas y destructivas, tales como el empleo de la fuerza física, la agresividad verbal, la venganza o la evitación” (Ibarrola y García, 2012, p. 23). Tales palabras explican que la detección tardía 
de un conflicto y no proceder a una resolución adecuada lo antes posible puede derivar en experiencias negativas.

En lo relativo a la solución, autores como Albaladejo, Ferrer, Reig y Fernández (2013) defienden la utilización de los conflictos que surgen en el aula para explicar a sus alumnos la manera de encontrar un acuerdo desde una perspectiva creativa y sencilla. Estas soluciones se pueden encontrar a través de estrategias de resolución de conflictos como la mediación y la consistencia.

"La mediación escolar no solo es una estrategia de resolución de conflictos, sino que conlleva una serie de valores y procedimientos que educan en la cultura de la Paz y consolidan formas de actuaciones y gestión de los conflictos profundamente participativas y democráticas" (Prada, 2008, p. 105). Esta manera de intervenir en la confrontación propicia que los alumnos establezcan una comunicación relajada expresando sus sentimientos y empatizando con su compañero.

La mediación entre iguales necesita la transmisión de un mensaje consistente por parte del maestro, que deberá ser coherente y consecuente para conseguir armonía dentro de su aula. Además, permite que el alumnado pronostique el grado del conflicto. Según Tattum (citado en Fernández, 2010):

\footnotetext{
"La inconsistencia en la aplicación de las reglas que se hayan marcado dentro del aula precipita los enfrentamientos. Esta inconsistencia puede ocurrir con rasgos tan sutiles como cuando un profesor reacciona de forma diferenciada hacia alumnos diferentes. Estos lo perciben como un símbolo de injusticia y falta de fiabilidad" (p.13).
}

Estas estrategias clásicas de resolución de conflictos están enfocadas a que, a largo plazo, ayuden a los pequeños a adquirir una mayor implicación y autonomía a la hora de proteger la vida en común (Ibarrola- García, 2012).

\section{EL JUEGO COMO RECURSO CONCILIADOR}

Tras un breve acercamiento a diversas definiciones sobre los conflictos, sus principales características y su relación con el ámbito educativo, resulta importante conocer la importancia que tiene el juego en la resolución de estas confrontaciones.

El juego es una destreza presente en todos los individuos. Aunque mantiene un fuerte vínculo con el ámbito infantil, dicha actividad puede ocurrir en cualquier momento de la vida (Chamorro, 2010).

Desde un punto de vista filosófico, Patierno (2016) opina que el juego "es visto por el pensamiento racional propio de la modernidad como una mera broma, como un modo de experimentar la ficción, o en términos más explícitos, como una mentira" (p. 5). Dicha definición entiende el juego como algo fantástico e irreal, cuya motivación principal es el ocio de relacionarse con los demás. El padre del psicoanálisis, Freud (citado en López, 2010), afirmó que el juego se debe a la necesidad de complacer los 
estímulos inconscientes de naturaleza amorosa y violenta, con el fin de transmitir los sentimientos, experiencias y emociones que genera. Es una herramienta que facilita la eliminación de las confrontaciones y ayuda a encontrar una solución mediante la imaginación.

Meneses y Monge (2001) también conciben el juego como transmisor de sentimiento, pues opinan que el maestro utiliza el juego para el desarrollo social de sus alumnos. Los autores afirman que, gracias a esta útil herramienta, los niños pueden desarrollar sensaciones de alegría, tristeza, miedo o enfado, entre otras muchas emociones que contribuyen al desarrollo integral del alumno.

Una concepción general sobre el juego la encontramos en Baena y Ruiz (2016), que dicen que ayuda a pulir aspectos de la personalidad del alumno. También le permite adquirir aprendizajes comunicativos, físicos y motores, facilitando la aparición de experiencias que favorecen al infante a la hora de desenvolverse en el mundo que le rodea.

Autores como Pol, Fuste, Martín, Palou y Masnou (2007) entienden esta herramienta como un proceso relacional espontáneo en el que los niños pueden cambiar sus normas mediante el diálogo, llegando a un acuerdo con los demás. Resulta importante ensalzar que lo importante no es el resultado, sino el correcto desarrollo del juego a través del entendimiento entre compañeros.

En relación a la resolución de conflictos que protagoniza este trabajo, encontramos la idea de los autores Girard y Koch (2001), que destacaron el juego de roles como, por ejemplo, el teatro para permitir que los alumnos observen las conductas inadecuadas desde otra perspectiva. Esta herramienta también resulta interesante para facilitar al alumno el propio reconocimiento de su comportamiento y la adquisición de destrezas.

Otro ejemplo de actividades que pueden servir para resolver conflictos, lo encontramos en los juegos de tipo cooperación-oposición. Dichas actividades consisten en el enfrentamiento entre dos equipos, dentro de los cuáles sus miembros deberán cooperar para vencer al rival. Pero este concepto de rivalidad no la comparten autores como Sáez, Lavega, Largardera, Costes y Serna (2014), pues señalan que el principal objetivo del juego es cambiar la idea inicial que tenemos de los jugadores adversarios. Es decir, verlo como un agente que trata de ponernos a prueba, no como un rival. Actividades de este tipo, además de fomentar la cooperación, enseñan a los alumnos un alto nivel de competencias comunicativas y emocionales, que facilitan la adaptación de los niños con su entorno, admitiendo de manera positiva la victoria del equipo contrincante.

La actividad que también puede ayudar a entender los conflictos es el conocido como juego de simular peleas o juego rudo, muy común entre los niños de edad infantil. En dicha actividad simbólica, los participantes simulan peleas que acaban con el dominio de uno sobre otro, comprendiendo que la 
fuerza puede ser inofensiva si se controla. Según Ruiz (2009, p. 104), "se trata de aprender a medir el poder propio y el ajeno y llegar a valorar la importancia de la negociación".

En general, todas estas aportaciones coinciden en la importancia que tiene el juego en el desarrollo de los pequeños, especialmente como elemento socializador y cooperativo.

\section{JUEGO COOPERATIVO}

Como afirma Jaqueira et al. (2013), "los juegos cooperativos son recursos magníficos para promover la educación de la convivencia pacífica y desencadenar emociones positivas intensas” (p. 18). Esta forma de ver los juegos cooperativos ya la expuso Llopis (2003), que afirmó que su práctica favorece la obtención de valores para todo el grupo, además de fomentar la comunicación entre sus miembros, a la hora de compartir sus experiencias, y empatizar con el compañero.

Autores como Cuéllar y Pérez (2015) analizaron varios estudios en base al vínculo existente entre el juego cooperativo y el desarrollo infantil, e indicaron que el aprendizaje cooperativo propicia aprendizajes sociales en alumnos con dificultades en su desarrollo.

Por su parte, Landazabal y Azumendi (2006) conciben el juego cooperativo como una actividad que facilita a los integrantes de un grupo a resolver un objetivo común. Cada miembro del equipo se ofrece ayuda mutuamente, ya que la finalidad que pretenden conseguir es la misma. Respaldando esta teoría, Aronson (citado en Orlick y López, 1995) subraya "la importancia de que todos los niños tengan la experiencia de ser miembros valiosos del grupo". (p. 242)

Además, también incluye mejoras físicas. Cilla y Omecaña (2007) estudiaron la actividad cooperativa como un recurso útil para que todos los participantes indaguen con su cuerpo y sus posibilidades de acción, proporcionando situaciones de aprendizaje que fomentan el movimiento y favorecen la destreza motriz.

\section{METODOLOGÍA}

En cuanto a la metodología de este trabajo, partimos de un aprendizaje significativo y constructivista, cuyo elemento principal es la comunicación de los protagonistas de esta propuesta de mejora educativa, los alumnos de segundo ciclo de infantil.

El proyecto está compuesto por una serie de actividades, complementarias a la transmisión de la enseñanza básica, en las que el docente observará y analizará las conductas de los infantes para, posteriormente, evaluarlas. Dichas actividades serán conducidas por el profesor, aunque no todas dependen de su supervisión y dirección, ya que se combinan juegos autónomos y dirigidos. 
El profesor, que se encargará de coordinar el conjunto de sesiones, irá registrando los cambios que va notando en los niños de manera diaria. De este modo, contará con información detallada y progresiva que le facilitará la posterior evaluación de sus alumnos, percibiendo el cambio experimentado por los niños desde que comenzaron el proceso, valorando si han mejorado su capacidad empática, sus relaciones con los demás o su madurez personal. En definitiva, cómo han reconducido y, por tanto, solventado sus problemas, analizando si han adquirido estrategias de resolución, como el diálogo entre ellos para superar las distintas situaciones adversas que se les proponen en el juego.

Las actividades incluidas en el proyecto son grupales, pues se pretende la mejora de la cooperatividad y el bienestar del alumnado. Conforme avance el plan, surgirán conflictos entre los niños y, ellos mismos, tendrán que ver el modo de resolverlo. Dichas actividades, se desempeñarán tanto en el lugar donde se imparten las clases diariamente como en el aula de psicomotricidad.

Los grupos estarán determinados por las conductas que los alumnos muestren en clase, mezclando a los que tengan comportamientos opuestos. De esta manera, se pretende favorecer la convivencia en el aula. Por ejemplo, a los alumnos que no manifiesten un comportamiento adecuado en clase se les ubicará con los niños más tranquilos, acostumbrados a ofrecer una conducta positiva y respetuosa. Esto provocará un clima en el que los alumnos alimenten sus capacidades de manera recíproca.

Además de los distintos espacios tradicionales que podemos encontrar en un aula de infantil, como el rincón de las matemáticas, lectoescritura, cuentacuentos o juegos, hemos propuesto la creación de un novedoso rincón de resolución de conflictos denominado 'Escucha y Habla', en el que los niños podrán solucionar sus diferencias, originadas o no dentro del aula. Para ello, los alumnos enemistados se sentarán frente a frente y, durante un tiempo limitado, tendrán la oportunidad de explicar su malestar y manifestar sus sentimientos de manera mutua. Con esto, se pretende enseñar a los alumnos a resolver sus conflictos de manera autónoma, sin que la figura del maestro tenga que mediar en sus problemas.

Tras la realización de las actividades incluidas en la propuesta didáctica, el docente formulará preguntas a sus alumnos, orientadas a conocer cómo se sienten, si les ha gustado la actividad, si consideran que han mejorado la relación con sus compañeros, si han sabido llegar al final del problema juntos, cómo lo han logrado... con la finalidad de despertar en los pequeños una capacidad de análisis, razonamiento y comunicación que les ayude a entender mejor lo ocurrido y a resolver sus conflictos.

En definitiva, como hemos manifestado en el apartado de Justificación, con este proyecto interdisciplinar que, abarca una variedad de ramas de Educación Infantil, pretendemos que el alumnado empiece a conocer, de manera independiente, su cuerpo y mente para mejorar su capacidad de resolución de conflictos a partir de un conjunto de principios y estrategias metodológicas flexibles y motivadoras, 
que ayudarán a los infantes a potenciar su personalidad a través de la impartición de distintas sesiones lúdicas y creativas.

\section{CONTENIDOS}

Como ya hemos avanzando, tanto la resolución de conflictos como el juego son dos conceptos fundamentales en el aula de Educación Infantil. Para llevar a cabo la primera debemos prestar atención a la segunda y viceversa, por lo que resulta fundamental relacionar ambas para la realización de este proyecto. Entre los contenidos que pretendemos que adquieran los niños tras la realización del curso-etapa encontramos los siguientes:

- Relación entre resolución de conflictos y juego.

- Observación de las distintas situaciones conflictivas.

- Adquisición de estrategias en la resolución de conflictos.

- Fomento de la cooperatividad y bienestar en el aula.

- Adquisición de técnicas comunicativas, competencias para la interacción entre el alumnado.

- Respeto a la diversidad dentro y fuera del aula, tolerando las diferencias físicas e intelectuales.

- Confección de su comportamiento, a través de actividades y juegos.

- Estimación de las directrices del aula.

- Evaluación de la adecuada conducta y reconocimiento de la misma.

- Ejercitación de su capacidad de pensamiento antes de actuar.

- Manifestación de malestar, sentimientos y emociones.

- Competencias orientadas a la correcta convivencia.

- Mejora de la conducta en el aula.

\section{PROPUESTA METODOLÓGICA}

\section{TEMPORALIZACIÓN Y ACTIVIDADES}

\subsection{TEMPORALIZACIÓN}

Una vez ideadas las actividades, que comentaremos en apartados posteriores, el docente debe proceder a realizar la programación del proyecto.

Distribuirá el trabajo en sesiones repartidas entre los cinco días lectivos con los que cuenta una semana. La propuesta se llevará a cabo a mediados del primer trimestre, para poder observar cómo van evolucionando los niños a raíz de dichas actividades, y así poder sacar sus propias conclusiones al final del ciclo escolar. Cada sesión durará sobre 40-50 minutos. 
La fase inicial del proyecto comenzará con una pequeña introducción al tema de resolución de conflictos, con un cuento denominado 'El búho y la ardilla'. Esta actividad motivadora conviene realizarla en momentos estratégicos, como pueden ser el comienzo del día o la vuelta de los niños a clase tras el recreo. Aunque, preferiblemente, la realizaremos el lunes a primera hora de la jornada escolar. La sesión durará cuarenta minutos. Los diez minutos restantes se dedicarán a comentar la fábula. El maestro formulará preguntas y, para finalizar, los niños dibujarán aquello que más les haya llamado la atención sobre la fábula.

Para complementar la fase inicial de la propuesta metodológica, realizaremos un Scape Room en el aula. En dicha sesión trabajaremos la educación en valores y la importancia de la actividad cooperativa como tarea fundamental para conseguir un objetivo común. Esta competencia se impartirá mediante un juego de escape denominado 'Las mesas amistosas' con cuatro actividades divididas entre los grupos del aula (equipo rojo, azul, amarillo y verde). Dichas actividades se realizarán en mesas rotatorias por las pasarán todos los grupos. Si el grupo tiende a cooperar los unos con los otros, conseguirán la llave que llega a abrir la caja misteriosa. Se realizará después de la asamblea que se hace todas las mañanas al entrar al aula, debido a que es uno de los mejores momentos de la jornada, en el que los niños prestan más atención. Esta sesión de Scape Room durará 45 minutos y la desarrollaremos el segundo día de la semana.

Durante la fase intermedia, desarrollaremos dos sesiones:

La primera sesión estará compuesta por dos actividades. Una de ellas, recibe el nombre de 'Cadena de gatitos para encontrar la bola de pelo'. Una vez finalizado dicho juego llevaremos a cabo el siguiente, denominado ‘¿Qué barco llegará antes a la isla perdida?’. Estas dos actividades son juegos cooperativos, en los que los alumnos tendrán que colaborar para llegar a una meta. Las actividades durarán 20 minutos cada una y se realizarán en las últimas horas de clase ya que son juegos que no requieren un alto grado de atención, sirven para desconectar y salir del aula.

La otra sesión perteneciente a la fase intermedia recibe el nombre de 'Descubre el objeto a través de la canción'. Por medio de una canción y con la implicación de los alumnos, el niño protagonista debe encontrar un objeto personal que sus compañeros han escondido por el aula. Este juego se realizará después del recreo, aprovechando el grado de actividad presente en los pequeños.

La última fase o fase de cierre, se llevará a cabo en la asamblea y serán partícipes todos los alumnos. Se realizará en torno a lo sucedido durante el recreo, debido a que es el lugar en el que se manifiestan más confrontaciones entre los alumnos. Por ese motivo, la asamblea posterior al recreo constituye el momento idóneo para tratar dichos temas. Para ello, diseñamos una actividad denominada 'El dado', que trata de realizar un pequeño repaso a los contenidos propuestos a lo largo de este proyecto. 
Tras esta breve introducción de las actividades propuestas en el proyecto, en el apartado siguiente las desarrollaremos más a fondo.

\subsection{ACTIVIDADES}

Como hemos avanzado en el apartado anterior, las propuestas didácticas incluidas en este proyecto estarán divididas en cinco sesiones. Contamos con una amplia diversidad de juegos y recursos muy útiles con los que podemos trabajar la resolución de conflictos y, en menor medida, el trabajo en equipo. Estas actividades las hemos dividido en 3 fases:

\subsection{FASE INICIAL}

Esta fase, dividida en dos sesiones, ayudará a los alumnos a comprender el concepto de conflicto.

Sesión 1. Narración del cuento 'El búho y la ardilla'

La finalidad de esta primera sesión es iniciar a los niños en el tema por excelencia de este proyecto: los conflictos. Para ello, contaremos un cuento relacionado con esta situación. La historia relata la conflictiva convivencia de un búho y una ardilla que, debido a sus diferencias, son incapaces de evitar las confrontaciones en su día a día en el bosque. El cuento finaliza con una bonita reconciliación, que muestra cómo podemos resolver conflictos utilizando el diálogo, llegando incluso a entablar una bonita amistad.

La narración de esta historia irá acompañada de las figuras de los protagonistas, realizadas previamente por la maestra. Para relatar la historia, se deberá leer previamente el cuento y utilizar los recursos necesarios para captar la atención de los niños. En definitiva, hacer nuestra la historia y contagiar curiosidad e interés por la fábula.

Para contagiar esta idea entre los alumnos, colocaremos el siguiente mensaje en las paredes del aula: "Leer un cuento es un momento mágico, nada ni nadie nos puede molestar". Con esto se pretende que los alumnos aprendan que hay situaciones en las que se deben evitar las interrupciones.

Posteriormente, cuando finalice la historia se realizarán preguntas para observar si los alumnos han comprendido el cuento, preguntas como las siguientes:

¿Cómo se llamaban los personajes?

¿Dónde viven el búho y la ardilla?

¿Cómo han resuelto el problema el búho y la ardilla?

¿Has vivido una situación parecida en el aula como la del búho y la ardilla?

¿Cómo se ha sentido el árbol al ver que la ardilla y el búho andaban todo el día peleándose?, ¿qué les ha dicho? 
Imaginad cómo se siente el árbol cuando el búho y la ardilla no paran de discutir.

Para terminar, hablaremos con los alumnos para hacer hincapié en la resolución de conflictos, dejando un espacio para que los niños dibujen lo que más les ha llamado la atención del cuento. Posteriormente, colgaremos sus dibujos en el aula.

\subsection{FASE INTERMEDIA O DE IMPLEMENTACIÓN}

En esta segunda fase, dividida también en dos sesiones, se buscará la unión del grupo mediante la cooperación y la consecución de objetivos comunes. Así como la respuesta positiva en contextos de competitividad.

\section{Sesión 3: 'Somos un equipo'}

En esta sesión vamos a realizar dos actividades en el aula de psicomotricidad, entre las que destacan:

Actividad 1. 'Cadena de gatitos para encontrar la bola de pelo'

El juego se realizará en un espacio abierto como, por ejemplo, el patio. Previamente diseñaremos un recorrido y dividiremos a los alumnos en dos equipos. Los niños tienen que ingeniárselas para formar una cadena a gatas, cogiendo el pie al compañero de delante. Pero no se pueden soltar, el alumno que se libere tendrá que volver al principio del juego. Finalmente, como he dicho, gana el equipo que termine el recorrido sin soltarse y se haga con la bola de pelo, simbolizada a través de un rollo de lana.

Actividad 2. ‘QQué barco llegará antes a la isla perdida?'

La actividad consiste en dividir a los alumnos en grupos de cinco. Cada equipo contará con un aro que simula la figura de un barco. Los alumnos deberán avanzar pegados dentro del aro sin que se les caiga. El equipo deberá coordinarse para llegar a su destino, una isla desierta dibujada en el suelo por la profesora. El grupo ganador no será el que antes llegue a la isla, sino el que haya realizado el recorrido de manera cooperativa y sin crear situaciones conflictivas.

Como en la actividad presente en la mesa dos de la sesión dedicada al Scape Room, los alumnos deberán demostrar, de manera conjunta, su capacidad de pensamiento antes de actuar.

\section{$\underline{\text { Sesión 4. 'Descubre el objeto a través de la canción' }}$}

Durante la realización de la asamblea se escogerá a un alumno para que inicie el juego. El niño tiene que salir fuera de clase para que los demás compañeros oculten en un rincón del aula un objeto personal del protagonista. Cuando el alumno vuelva a entrar al aula, sus compañeros le advertirán que un objeto suyo se encuentra escondido, pero que entre todos le ayudarán a encontrarlo. Para ello, le cantarán la siguiente canción: 
"Escondido está, escondido está presta atención a través de la canción y lo encontrarás".

La intensidad con la que sus compañeros canten la canción le indicará al alumno lo cerca que se encuentra de su objeto. Por ejemplo: si el objeto está muy lejano, sus compañeros le cantarán la canción con un tono. Pero si, por el contrario, el pequeño se encuentra cerca del objeto, los alumnos cantarán con más intensidad.

Una vez que el protagonista ha recuperado su objeto, los demás alumnos le felicitaran. El niño, lejos de enfadarse, deberá agradecerles a sus compañeros la ayuda, pues sin la colaboración de todos no lo habría conseguido. Con actividades como esta, los alumnos potencian valores tan necesarios para la resolución de conflictos como la amabilidad y la ayuda.

\subsection{FASE DE CIERRE}

En esta última fase más reflexiva, comprobaremos si a los alumnos les ha quedado claro lo practicado a lo largo de la semana. Si todavía tienen la idea equivocada de resolución de conflictos a través de la violencia física y verbal o si, por el contrario, son capaces de utilizar la diversión que les proporciona el juego para razonar sobre lo sucedido y solucionar sus confrontaciones. En definitiva, esta última fase nos sirve para comprobar si el juego es una buena herramienta para la resolución de conflictos.

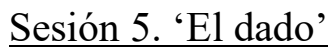

Durante esta sesión, se sentará a los alumnos en forma de círculo alrededor de un dado gigante. El maestro escogerá a un alumno para lanzar el dado, diseñado previamente por él profesor. El dado no será un dado clásico con números, sino que cada una de sus caras reflejará diferentes situaciones conflictivas y amistosas presentes en la vida cotidiana.

Entre las ilustraciones que podemos encontrar en las seis caras del dado, se encuentran: un niño gritando a sus padres, pequeños marginando a un compañero durante un juego o dos niños abrazándose, entre otros.

Por lo tanto, el alumno lanzará el dado y se le presentará una ilustración. Luego, tendrá que explicar la viñeta a sus compañeros, razonando si se trata de una situación positiva o negativa, en cuyo caso tendrá que aportar una manera de resolverla. Por ejemplo, tendrá que reflexionar sobre los motivos que han llevado a los personajes de la ilustración a abrazarse o a discutir. 
Durante el desarrollo de la actividad, el maestro deberá anotar lo que piensan y ven los niños en un mural grande. Posteriormente, el profesor deberá leer todo lo que han dicho delante de sus alumnos. Para esta actividad es muy importante que dejemos que los niños se expresen y piensen libremente.

\section{CONCLUSIÓN}

La elección de esta línea de TFG estuvo motivada en la dificultad que me encontré al inicio de mis prácticas curriculares. Me sentía muy desinformada y pensaba que era muy complicado resolver conflictos entre mis alumnos. Entonces, investigué sobre técnicas y estrategias de resolución de conflictos y las puse en práctica en mi aula de infantil. El resultado fue sorprendente y, debido al éxito que tuvo su aplicación, decidí seguir formándome en este asunto, para llevarlo a la práctica en el futuro y conseguir una clase libre de enfrentamientos.

Por lo tanto, puedo decir que revisar la opinión de distintos autores me ha ayudado a buscar soluciones. He comprobado que no hay que ver los conflictos como algo negativo, sino como una oportunidad educativa para los alumnos de educación infantil. Como maestra, intentaré enfocar el tema de esta manera en mi trabajo con los pequeños.

Consideramos que la propuesta educativa expuesta en este TFG es útil y beneficiosa a la hora de propiciar la cooperación entre los alumnos y la unidad del aula. Pensamos que, actualmente, es una jornada que podría llevarse a cabo en las escuelas, coordinándose con otros proyectos presentes en el sistema educativo, como el ciclo del agua, las manos, la vida sana o las estaciones del año. También estimamos que este proyecto semanal conservaría su utilidad implantándose en periodos de tiempo más largos, de manera mensual o trimestral.

Para llevar a cabo el proyecto necesitamos espacios adecuados y definidos, como es el aula de psicomotricidad o el patio. En esta mejora didáctica, se propone una idea que atiende a la figura de los niños, los protagonistas para realizar dichas actividades dentro del horario escolar. Además, ensalza la implicación de los alumnos, ya desde el ciclo infantil, en la participación como algo indispensable para su educación.

La principal motivación de este trabajo ha sido ver los conflictos como una experiencia atractiva y dinámica, pensando que por medio de ellos y de forma lúdica los niños pueden aprender distintas estrategias de resolución de conflictos. En un principio puede existir cierto desorden en el aula, pues se necesitan grandes recursos y saber dar buenas soluciones en el tiempo necesario para conseguir lo que pretendemos enseñarles. 
La implantación de innovaciones requiere de dedicación y esfuerzo. Muchos maestros prefieren trabajar de forma tradicional, pero las nuevas promociones queremos obtener ventajas y conseguir objetivos haciendo que los alumnos interactúen entre sí. 


\section{BIBLIOGRAFÍA}

Albaladejo-Blázquez, N., Ferrer-Cascales, R., Reig-Ferrer, A., \& Fernández-Pascual, M. D. (2013). ¿Existe violencia escolar en Educación Infantil y Primaria?: una propuesta para su evaluación y gestión. Anales de psicología, 29(3), $1060-1069$. Recuperado de https://revistas.um.es/analesps/article/view/analesps.29.3.158431/152231

Chillón, G. D (1996). Tratamiento de los valores en la educación infantil. Revista interuniversitaria de formación del profesorado, (27), 21-23. Recuperado de file:///C:/DialnetTratamientoDeLosValoresEnLaEducacionInfantil$117914 \% 20(1) . p d f$

Chamorro, I. L. (2010). El juego en la educación infantil y primaria. Autodidacta, 1(3), 19-37. Recuperado de http://educacioninicial.mx/wp-content/uploads/2017/11/JuegoEIP.pdf

Cilla, R. O., \& Omeñaca, J. V. R. (2007). Juegos cooperativos y educación física. Editorial Paidotribo. Recuperado de https://books.google.es/books?hl=es\&lr=\&id=fy_qy1n84H8C\&oi=fnd\&pg=PA5\&dq=Juegos+cooperativos+y+educaci\%C3 $\% \mathrm{~B} 3 \mathrm{n}+\mathrm{f} \% \mathrm{C} 3 \%$ ADsica.++cilla+\&ots=VBqMzgciEx\&sig=uBPbjYj7J3cGfNDu0enyKop_094\#v=onepage\&q=Juegos \%20coo perativos $\% 20 \mathrm{y} \% 20$ educaci\%C3\%B3n $\% 20 \mathrm{f} \% \mathrm{C} 3 \%$ ADsica. $\% 20 \% 20$ cilla\&f=false

Cuéllar de Lucas, Y., Pérez-Brunicardi, D., \& De la Iglesia, M. (2015). Enseñanza cooperativa como instrumento para la inclusión de un niño con Trastorno de Espectro Autista (TEA) mediante juegos cooperativos en un aula de Educación Infantil. Revista Arbitrada del CIEG-Centro de Investigación Y Estudios Gerenciales, 21, 259-271. Recuperado de http://www.grupocieg.org/archivos_revista/Ed.\%2021\%20(259-

271)\%20Cu\%C3\%A911ar\%20de\%20Lucas\%20y\%20otros\%20-\%20septiembre\%202015_articulo_id213.pdf

De Pol, C. T., Fusté, S., Martín, L., Palou, S., \& Masnou, F. (2007). Jugando para vivir, viviendo para jugar: el juego como motor del aprendizaje. Planificar la etapa 0-6: compromiso de sus agentes y práctica cotidiana, 21, 127. Recuperado de https://books.google.es/books?hl=es\&lr=\&id=s-

B_kqRnPxwC\&oi=fnd\&pg=PA127\&dq=Jugando+para+vivir,+viviendo+para+jugar:+el+juego+como+motor+del+aprendiza je\&ots $=91 x S \_k 2 Z 3-$

\&sig=GbOomyvuP2TL42u_01sZTzQpUj0\#v=onepage\&q=Jugando\%20para\%20vivir\%2C\%20viviendo\%20para\%20jugar\% $3 \mathrm{~A} \% 20 \mathrm{el} \% 20 \mathrm{juego} \% 20$ como $\% 20$ motor $\% 20 \mathrm{del} \% 20$ aprendizaje $\& \mathrm{f}=$ false

De Prada, J., \& López, J. (2008). La mediación como estrategia de resolución de conflictos en el ámbito escolar. Documentación $\quad$ social, 148, $\quad 99-116 . \quad$ Recuperado de http://convivejoven.semsys.itesi.edu.mx/cargas/Articulos/LA\%20MEDIACI\%C3\%93N\%20COMO\%20ESTRATEGIA\%20 DE\%20RESOLUCI\%C3\%93N\%20DE\%20CONFLICTOS\%20EN\%20EL\%20\%C3\%81MBITO\%20ESCOLAR.pdf

Extremera, A. B., \& Montero, P. J. R. (2016). El juego motor como actividad física organizada en la enseñanza y la recreación. EmásF: revista digital de educación física, (38), 73-86. Recuperado de https://dialnet.unirioja.es/servlet/articulo?codigo=5351993

Fernández, I. (2010). Prevención de la violencia y resolución de conflictos. Narcea Ediciones. Recuperado de https://s3.amazonaws.com/academia.edu.documents/46790287/40FERNaNDEZ-Isabel-cap-4-Tipos-de-hechos-

violentos_1.pdf?AWSAccessKeyId=AKIAIWOWYYGZ2Y53UL3A\&Expires=1559173461\&Signature=uBcHKDPilKpelJ9

PGndeUVKN3Kg\%3D\&response-content-

disposition=inline\%3B\%20filename\%3DPrevencion_de_la_violencia_y_resolucion.pdf

8

Girard, K., \& Koch, S. J. (2001). Resolución de conflictos en las escuelas: manual para educadores. Ediciones Granica SA. Recuperado https://books.google.es/books?hl=es\&lr=\&id=dvYZg3vXg34C\&oi=fnd\&pg=PA19\&dq=Resoluci\%C3\%B3n+de+conflictos+ en+las+escuelas:+manual+para+educadores.\&ots=71Gun7XxE0\&sig=58se4CwsgLGy1_f7t9YUDyka7pk\#v=onepage\&q=Re soluci\%C3\%B3n\%20de\%20conflictos\%20en\%20las\%20escuelas $\% 3 \mathrm{~A} \% 20$ manual $\% 20$ para $\% 20$ educadores.\&f=false

García, I. F. (1998). Prevención de la violencia y resolución de conflictos: el clima escolar como factor de calidad (Vol. 142). Narcea Ediciones. Recuperado de https://books.google.es/books?hl=es\&lr=\&id=zOsRnNJ_9BAC\&oi=fnd\&pg=PA11\&dq=fernandez+dimension+escolar+conf lictos\&ots=K181Xcu83w\&sig=vtJj9e6xuPVrgVelHQeUjmI6CH4\#v=onepage\&q=fernandez\%20dimension\%20escolar\%20c onflictos $\& \mathrm{f}=$ false 
Hamodi Galán, C., \& Jiménez Robles, L. (2018). Modelos de prevención del bulling: ¿ qué se puede hacer en educación infantil? . IE Revista de investigación educativa de la REDIECH, 9(16), 29-50. Recuperado de http://www.scielo.org.mx/scielo.php?pid=S244885502018000100029\&script=sci_arttext

Ibarrola-García, S., \& Redín, C. I. (2012). La convivencia escolar en positivo: mediación y resolución de conflictos. Pirámide. Recuperado de https://revistas.comillas.edu/index.php/padresymaestros/article/download/8597/8156

Jaqueira, A. R., Lavega Burgués, P., Lagardera Otero, F., Araujo, P., \& Rodrigues, M. (2013). Educando para la paz jugando: género $\mathrm{y}$ emociones en la práctica de juegos cooperativos competitivos. Recuperado de https://revistas.um.es/educatio/article/download/194071/159431/

Landazabal, M. G., \& Azumendi, J. M. F. (2006). El juego cooperativo para prevenir la violencia en los centros escolares: evaluación de programas de intervención para la educación infantil, primaria y secundaria (Vol. 4). Ministerio de Educación. Recuperado

https://books.google.es/books?hl=es\&lr=\&id=QxuyWt0uu6kC\&oi=fnd\&pg=PA7\&dq=El+juego+cooperativo+para+prevenir +la+violencia+en+los+centros+escolares:+evaluaci\% $3 \% \mathrm{~B} 3 \mathrm{n}+\mathrm{de}+$ programas+de+intervenci\%C3\%B3n+para+la+educaci\% $\mathrm{C} 3 \% \mathrm{~B} 3 \mathrm{n}+\mathrm{infantil},+$ primaria+y+secundaria+\&ots=Ey4HK3Fj5A\&sig=Qd78Ny0OhC_157CUstHLFnpu05I\#v=onepage\&q= El\%20juego\%20cooperativo\%20para\%20prevenir\%20la\%20violencia\%20en\%20los\%20centros\%20escolares\%3A\%20evalu aci\%C3\%B3n\%20de\%20programas\%20de\%20intervenci\%C3\%B3n\%20para\%20la\%20educaci\%C3\%B3n\%20infantil\%2C $\% 20$ primaria $\% 20 \mathrm{y} \% 20$ secundaria\&f=false

Lapponi, S. F. (2013). Resolución de conflictos en la escuela: una herramienta para la cultura de paz y la convivencia. Contextos Educativos. Revista de Educación, 91-106. (3), Recuperado de https://publicaciones.unirioja.es/ojs/index.php/contextos/article/view/466/430

Llopis, C. (2003). Los derechos humanos en educación infantil: cuentos, juegos y otras actividades (Vol. 50). Narcea Ediciones. Recuperado de https://books.google.es/books?hl=es\&lr=\&id=v5yIkiEFlzAC\&oi=fnd\&pg=PA9\&dq=Los+derechos+humanos+en+educaci\% C3\%B3n+infantil:+cuentos,+juegos+y+otras+actividades+\&ots=EfHxUhHwzn\&sig=MXpU151Le5y8AIuxYDsTIWuxkp0\#v $=$ onepage $\& q=$ Los $\% 20$ derechos $\% 20$ humanos $\% 20$ en $\% 20$ educaci $\% \mathrm{C} 3 \% \mathrm{~B} 3 \mathrm{n} \% 20$ infantil $\% 3 \mathrm{~A} \% 20$ cuentos\%2C $\% 20 \mathrm{juegos} \% 20$ y\%20otras\%20actividades\&f=false

Marín-Díaz, V., \& Sánchez-Cuenca, C. (2015). Formación en valores y cuentos tradicionales en la etapa de educación infantil. Revista Latinoamericana de Ciencias Sociales, Niñez y Juventud, 13(2), 1093-1106. Recuperado de https://www.redalyc.org/pdf/773/77340728038.pdf

Meneses Montero, M., \& Monge Alvarado, M. D. L. Á. (2001). El juego en los niños: enfoque teórico. Educación, 25(2). Recuperado de https://www.redalyc.org/html/440/44025210/

Moral, E. M. S., \& Martín-Albo, C. V. (2003). La Educación Intercultural y la educación en valores. Educación y futuro: revista de investigación aplicada y experiencias educativas, (8), 71-80. Recuperado de https://dialnet.unirioja.es/descarga/articulo/2044312.pdf

Narejo, N., \& Salazar, M. (2002). Vías para abordar los conflictos en el aula. Revista electrónica interuniversitaria de formación del profesorado, 5(4). Recuperado de file://C:/Users/Dialnet-ViasParaAbordarLosConflictosEnElAula-1034377\%20(1).pdf

Orlick, T., \& López, M. M. (1995). Libres para cooperar, libres para crear:(nuevos juegos y deportes cooperativos) (Vol. 2). Editorial paidotribo. Recuperado de https://books.google.es/books?hl=es\&lr=\&id=VMyn5SVi_4C\&oi=fnd\&pg=PA15\&dq=) + Libres + para+cooperar, + libres + para + crear:(nuevos+juegos $+\mathrm{y}+$ deportes + cooperativos $\&$ ots $=\mathrm{f} 1$ KO7r47jp\&sig=o20vuFqHAT4yaLKLVHDtaO_kqAA\#v=onepage\&q=).\%20Libres\%20para\%20cooperar\%2C\%20libres\%2 0para\%20crear\%3A(nuevos\%20juegos\%20y\%20deportes\%20cooperativos\&f=false

Patierno, N. (2016). El juego como estrategia de intervención para la resolución de conflictos en escuelas secundarias. Lúdicamente, 5(9). Recuperado de http://ppct.caicyt.gov.ar/index.php/ludicamente/article/view/7922/pdf

Ramos, J. D. (2017). Educación emocional para la resolución de conflictos en la escuela: una aproximación desde la educación social. Intervención psicoeducativa en la desadaptación social: IPSE-ds, (10), 11-22. Recuperado de https://dialnet.unirioja.es/descarga/articulo/6638825.pdf 
"Real Decreto 1630/2006, 29 de diciembre, de Educación (BOE num.185, 4 enero 2007)". Recuperado de https://www.boe.es/buscar/pdf/2007/BOE-A-2007-185-consolidado.pdf

Rodríguez, M. L. G., \& Ordóñez, G. B. (2017). Conflictos, mediación en la Escuela Infantil. Revista De Estudios E Investigación En Psicología Y Educación, 077-081. Recuperado http://revistas.udc.es/index.php/reipe/article/view/reipe.2017.0.02.2725/pdf

Rodríguez, M. A. S. (2004). El color de las emociones y el tratamiento del conflicto en el aula. Tabanque: Revista pedagógica, (18) 135-152. Recuperado de file:///C:/Dialnet-ElColorDeLasEmocionesYElTratamientoDelConflictoEnE-1138359.pdf

Ruiz, R. O. (2004) Lo mejor y lo peor de las redes de iguales: juego, conflicto y violencia. Revista Interuniversitaria de la formación del profesorado, (44), 93-113. Recuperado de https://dialnet.unirioja.es/servlet/articulo?codigo=249634

Sáez, U. N. A. I., Lavega, P., Lagardera, J., Costes, A., \& Serna, J. (2014). ¿ Por qué te peleas? Conflictos motores y emociones negativas en la clase de Educación Física: el caso de los juegos de oposición. Educatio Siglo XX1, 32(2), 71-90. Recuperado de https://revistas.um.es/educatio/article/view/194091/159451

Serrano, G. P., \& de Guzmán Puya, M. V. P. (2011). Aprender a convivir: el conflicto como oportunidad de crecimiento (Vol. 189). Narcea Ediciones. Recuperado de https://books.google.es/books?hl=es\&lr=\&id=V9V9IzZTMAC\&oi=fnd\&pg=PA7\&dq=).+Aprender+a+convivir:+el+conflicto+como+oportunidad+de+crecimiento\&ots=pwlbpv4 9xQ\&sig=Ts6u4OXksL6-

FYs7wPMZ7Jkjtao\#v=onepage\&q=).\%20Aprender\%20a\%20convivir\%3A\%20el\%20conflicto\%20como\%20oportunidad\%2 $0 \mathrm{de} \% 20$ crecimiento\&f$=$ false

Torrego, J. C., \& Galán, A. (2008). Investigación evaluativa sobre el programa de mediación de conflictos en centros escolares. $\begin{array}{llll}\text { Revista de 347(3), } & \text { Educación, } & \text { Recuperado 394. }\end{array}$ https://sede.educacion.gob.es/publiventa/descarga.action?f_codigo_agc=12407_19

Touriñán-López, J. M. (2006). La educación intercultural como ejercicio de educación en valores. Recuperado de https://dadun.unav.edu/bitstream/10171/8928/1/EA.PDF 